\title{
SELF-SIMILAR SETS WITH SUPER-EXPONENTIAL CLOSE CYLINDERS
}

\author{
Changhao Chen \\ The Chinese University of Hong Kong, Department of Mathematics \\ Shatin, Hong Kong; chench@ahu.edu.cn
}

\begin{abstract}
Baker (2019), Bárány and Käenmäki (2019) independently showed that there exist iterated function systems without exact overlaps and there are super-exponentially close cylinders at all small levels. We adapt the method of Baker and obtain further examples of this type. We prove that for any algebraic number $\beta \geqslant 2$ there exist real numbers $s, t$ such that the iterated function system

$$
\left\{\frac{x}{\beta}, \frac{x+1}{\beta}, \frac{x+s}{\beta}, \frac{x+t}{\beta}\right\}
$$

satisfies the above property.
\end{abstract}

\section{Introduction}

An iterated function system is a family $\Phi=\left\{\varphi_{i}\right\}_{i \in \Lambda}$ of finite contraction maps on $\mathbf{R}^{d}$. Hutchinson [11] proved that there exists a unique non-empty compact set $X \subset \mathbf{R}^{d}$ such that

$$
X=\bigcup_{i \in \Lambda} \varphi_{i}(X)
$$

We call $X$ the self-similar set of $\Phi$ if all $\varphi_{i}$ are similarity maps. One of the fundamental problems in fractal geometry is to determine the Hausdorff dimension of self-similar sets, see [6] for the definition and basic properties of Hausdorff dimension. In this paper we use $\operatorname{dim} E$ to denote the Hausdorff dimension of the set $E$.

There are various dimensions for measuring a given set, and their values may be different in general, see e.g. [6, 14]. However, Falconer [5] proved that self-similar sets always have equal Hausdorff and box dimensions.

For any self-similar set $X$, there is a trivial upper bound for $\operatorname{dim} X$. More precisely, let $\Phi=\left\{r_{i} x+t_{i}\right\}_{i \in \Lambda}$ with $0<\left|r_{i}\right|<1, t_{i} \in \mathbf{R}^{d}, i \in \Lambda$ and $\Lambda$ has finitely many elements. Let $\operatorname{dim}_{S} \Phi$ be the unique solution $s$ such that $\sum_{i \in \Lambda}\left|r_{i}\right|^{s}=1$, then we have, see [6, Chapter 9],

$$
\operatorname{dim} X \leqslant \min \left\{d, \operatorname{dim}_{S} \Phi\right\} .
$$

Hutchinson [11] proved that if $\Phi$ satisfies the open set condition then the equality holds in (1.1). We remark that there are many other separation conditions which are often added on $\Phi$, see e.g. [13, 4, 15] for more details.

We say that there is a dimension drop for $\Phi$ if the strict inequality holds in (1.1). It is not hard to show that the dimension drop occurs when $\Phi$ has sufficiently many exact overlaps, see [10].

For $\mathbf{i}=i_{1} \ldots i_{n} \in \Lambda^{n}$ denote $\varphi_{\mathbf{i}}=\varphi_{i_{1}} \circ \ldots \circ \varphi_{i_{n}}$. We say that $\Phi$ has exact overlaps if there exist $\mathbf{i}, \mathbf{j} \in \Lambda^{n}$ with $\mathbf{i} \neq \mathbf{j}$ such that $\varphi_{\mathbf{i}}=\varphi_{\mathbf{j}}$. Note that the set $\varphi_{\mathbf{i}}(X)$ is often called a cylinder of $\Phi$ or $X$. We remark that for $d=1$, so far the exact overlaps are

https://doi.org/10.5186/aasfm.2021.4646

2020 Mathematics Subject Classification: Primary 28A80, 11J70.

Key words: Self-similar sets, exact overlaps, continued fractions. 
the only known cause for the dimension drop. We have the following conjecture, see [17], and for the recent achievements, see [16, 18].

Conjecture 1.1. Let $\Phi$ be an IFS consisting of similarity maps on $\mathbf{R}$. If there is a dimension drop for $\Phi$, then $\Phi$ has exact overlaps.

Hochman [9] proved that if there is a dimension drop for $\Phi$ then there are superexponentially close cylinders. To be precise, following Hochman [10] we use the following notation to quantify the amount of overlaps. Define a distance $d(\cdot, \cdot)$ between any two similarities $\varphi(x)=a x+b$ and $\varphi^{\prime}(x)=a^{\prime} x+b^{\prime}$ by

$$
d\left(\varphi, \varphi^{\prime}\right)=\left|b-b^{\prime}\right|+|\log | a|-\log | a^{\prime}|| .
$$

For a given IFS $\Phi=\left\{\varphi_{i}\right\}_{i \in \Lambda}$ and $n \in \mathbf{N}$ denote

$$
\Delta_{n}=\min \left\{d\left(\varphi_{\mathbf{i}}, \varphi_{\mathbf{j}}\right): \mathbf{i}, \mathbf{j} \in \Lambda^{n}, \mathbf{i} \neq \mathbf{j}\right\}
$$

Note that there are exact overlaps if and only if $\Delta_{n}=0$ for some $n$. Moreover for any IFS $\Phi$ there exists $0<c<1$ such that $\Delta_{n} \leqslant c^{n}$ for all large enough $n$. Clearly there is an IFS $\Phi$ such that $\Delta_{n} \geqslant c^{n}$ for some $c>0$ and all $n \in \mathbf{N}$, for instance the generating IFS $\left\{\frac{1}{3} x, \frac{1}{3} x+\frac{2}{3}\right\}$ of the Cantor ternary set.

For an IFS $\Phi$ on $\mathbf{R}$ Hochman [9] proved that if

$$
\operatorname{dim} X<\min \left\{1, \operatorname{dim}_{s} \Phi\right\},
$$

then for any $c>0$ there exists $N$ such that for all $n \geqslant N$ one has

$$
\Delta_{n} \leqslant c^{n}
$$

We remark that this leads to many applications in the dimension theory of self-similar sets, see [9] for more details.

By the above result of Hochman and Conjecture 1.1, it is natural to ask whether an IFS $\Phi$ on $\mathbf{R}$ such that the estimate (1.3) holds (for any $c>0$ and all large enough $n$ ) would imply the IFS $\Phi$ has exact overlaps, see [10]. For this direction, Baker [1], Bárány and Käenmäki [3] independently showed that there exists an IFS $\Phi$ without exact overlaps and there are super-exponentially close cylinders at all small levels. More precisely, for any positive sequence $\left\{\varepsilon_{n}\right\}$ Baker [1], Bárány and Käenmäki [3] showed that there exists an IFS $\Phi$ without exact overlaps such that

$$
\Delta_{n} \leqslant \varepsilon_{n}, \quad \forall n \in \mathbf{N} .
$$

In this paper, by adapting the method of Baker [1] we obtain further examples of this type. The IFS of Baker is the form

$$
\begin{gathered}
\Phi_{s, t}=\left\{\varphi_{1}(x)=\frac{x}{2}, \varphi_{2}(x)=\frac{x+1}{2}, \varphi_{3}(x)=\frac{x+s}{2}, \varphi_{4}(x)=\frac{x+t}{2},\right. \\
\left.\varphi_{5}(x)=\frac{x+1+s}{2}, \varphi_{6}(x)=\frac{x+1+t}{2}\right\} .
\end{gathered}
$$

Precisely, Baker [1] showed that for any positive sequence $\left\{\varepsilon_{n}\right\}$ there exist $s, t \in \mathbf{R}$, such that the IFS $\Phi_{s, t}$ satisfies (1.4). Roughly speaking, Baker's arguments can be divided into three steps. Firstly, for the above IFS $\Phi_{s, t}$, there is a "nice" upper bound for $\Delta_{n}$ depending on the Diophantine properties of $s$ and $t$ (i.e., whether the numbers $s$ and $t$ can be well approximately by rational numbers). Secondly, using the properties of continued fractions (with integer elements), Baker [1] construct two numbers $s, t \in \mathbf{R}$ such that for the IFS $\Phi_{s, t}$ of (1.5) one has $\Delta_{n} \leqslant \varepsilon_{n}$ for all $n \in \mathbf{N}$. Thirdly, show that the IFS $\Phi_{s, t}$ satisfies the desired property. 
Baker [1, Remark 2.2] also showed that if we take the fraction $1 / 8$ instead of $1 / 2$ in (1.5), we can also find $s, t \in \mathbf{R}$ such that the IFS $\Phi_{s, t}$ satisfies (1.4). Moreover, [1, Remark 2.2] implies that we could take any other natural numbers $(\geqslant 2)$ instead of $1 / 2$ in (1.5) to obtain the desired IFS. Indeed this is our start point of this paper, by adapting the method of Baker [1] and mimicking his argument, we can take algebraic number instead of $1 / 2$ in (1.5) to obtain the desired IFS. We also use the above three steps of Baker, however for the non-integer case $\beta$ we will use the continued fractions with non-integer elements which is the main difference from [1].

Our contribution is that, except the previous examples of Baker [1], Bárány and Käenmäki [3], we provide further IFS without overlaps and there are superexponentially close cylinders at all small levels.

We consider the following variant IFS of (1.5),

$$
\Phi_{\beta, s, t}=\left\{\frac{x}{\beta}, \frac{x+1}{\beta}, \frac{x+s}{\beta}, \frac{x+t}{\beta}\right\} .
$$

For convenience we write $\Phi_{\beta, s, t}=\left\{\varphi_{i}\right\}_{i \in \Lambda}$ where $\Lambda=\{1,2,3,4\}$. Moreover, with respect to (1.2), for $n \in \mathbf{N}$ denote

$$
\Delta_{n}(\beta, s, t)=\min \left\{\left|\varphi_{\mathbf{i}}(0)-\varphi_{\mathbf{j}}(0)\right|: \mathbf{i}, \mathbf{j} \in \Lambda^{n}, \mathbf{i} \neq \mathbf{j}\right\} .
$$

Theorem 1.2. Let $\beta \geqslant 2$ be an algebraic number. Then for any positive sequence $\left\{\varepsilon_{n}\right\}$ there exists $\Phi_{\beta, s, t}$ without exact overlaps and

$$
\Delta_{n}(\beta, s, t) \leqslant \varepsilon_{n}, \quad \forall n \in \mathbf{N} .
$$

We remark that (in our setting) for any algebraic $\beta \geqslant 2$ and the self-similar set $X$ of the IFS $\Phi_{\beta, s, t}$ of Theorem 1.2, Rapaport [16] showed that

$$
\operatorname{dim} X=\min \left\{1, \frac{\log 4}{\log \beta}\right\} .
$$

We note that there are self-similar measures which are related to self-similar sets. The conjecture 1.1 can also be formulated to the self-similar measures as well, see $[1,10,16]$.

We remark that recently Baker [2] gives a more general construction such that his general example will contain the example in [1] and the example of this paper as well, moreover his construction allows for transcendental similarity ratios. However, we think that it is still meaningful to show the explicit construction of the example in the Theorem 1.2 by using the properties of continued fractions and algebraic numbers.

\section{Preparation}

2.1. Notation. Let $\beta \geqslant 2$ be a fixed algebraic number throughout the paper. Denote

$$
\begin{aligned}
\mathbf{P}_{\beta} & =\left\{\beta^{n}: n=0,1,2, \ldots\right\} \\
\mathbf{Z}_{\beta} & =\{f(\beta): f \in \mathbf{Z}[x]\} ; \\
\mathbf{Q}_{\beta} & =\left\{f / g: f, g \in \mathbf{Z}_{\beta}, g \neq 0\right\} .
\end{aligned}
$$

For a number $f(\beta) \in \mathbf{Z}_{\beta}$ we sometimes regard $f(\beta)$ as a polynomial with the indeterminate $\beta$ when there is no confusion. Thus the degree of $f(\beta)$ is understood as the degree of $f \in \mathbf{Z}[x]$. 
2.2. Iterated function system $\boldsymbol{\Phi}_{\beta, s, t}$. We first introduce the following " $\beta$ based" sets.

$$
\mathcal{B}_{n}=\left\{\sum_{j=1}^{n} \omega_{j} \beta^{j-1}: \omega_{j} \in\{0,1\}\right\} \quad \text { and } \quad \mathcal{B}=\bigcup_{n=1}^{\infty} \mathcal{B}_{n}
$$

Note that by the restriction $\beta \geqslant 2$ any element in $\mathcal{B}$ has a unique representation. Indeed this follows by the fact that for any $k \geqslant 1$ we have

$$
\beta^{k}>\beta^{k-1}+\ldots+1 \text {. }
$$

We remark that this is the reason of letting $\beta \geqslant 2$ in Theorem 1.2.

In analogy of Baker [1, Lemma 2.1] we have the following upper bound for $\Delta_{n}(\beta, s, t)$.

Lemma 2.1. For the IFS $\Phi_{\beta, s, t}$ and $n \in \mathbf{N}$ we have

$$
\Delta_{n}(\beta, s, t) \leqslant \beta^{-n} \min \left\{\min _{\substack{p, q \in \mathcal{B}_{n} \\(p, q) \neq(0,0)}}|q s-p|, \min _{\substack{p, q \in \mathcal{B}_{n} \\(p, q) \neq(0,0)}}|q t-p|\right\} .
$$

Proof. Observe that

$$
\left\{\varphi_{\mathbf{i}}(0): \mathbf{i} \in \Lambda^{n}\right\}=\left\{\sum_{j=1}^{n} c_{j} \beta^{-j+1}: c_{j} \in\{0,1 / \beta, s / \beta, t / \beta\}\right\} .
$$

Recall that the arithmetic sum of sets $X, Y \subseteq \mathbf{R}$ is defined as

$$
X+Y=\{x+y: x \in X, y \in Y\} .
$$

Moreover for $\rho \in \mathbf{R}$ denote $\rho X=\{\rho x: x \in X\}$. Let $A=\{0,1, s, t\}$ then

$$
\left\{\varphi_{\mathbf{i}}(0): \mathbf{i} \in \Lambda^{n}\right\}=\beta^{-n}\left(A+\beta A+\ldots+\beta^{n-1} A\right) .
$$

Applying (2.1) we derive

$$
\mathcal{B}_{n} \cup s \mathcal{B}_{n} \cup t \mathcal{B}_{n} \subseteq A+\beta A+\ldots+\beta^{n-1} A .
$$

Combining with (2.2) and (1.6) we obtain the desired bound.

Lemma 2.2. Let $\beta \geqslant 2$ and $s, t \notin \mathbf{Q}_{\beta}$. Suppose the IFS $\Phi_{\beta, s, t}$ has exact overlaps then there exit $f(\beta), g(\beta) \in \mathbf{Z}_{\beta} \backslash\{0\}$ and $h(\beta) \in \mathbf{Z}_{\beta}$ such that

$$
s=\frac{f(\beta)}{g(\beta)} t+\frac{h(\beta)}{g(\beta)} \text {. }
$$

Proof. Since $\Phi_{\beta, s, t}$ has exact overlaps, there is $n \in \mathbf{N}$ and $\mathbf{i}, \mathbf{j} \in \Lambda^{n}, \mathbf{i} \neq \mathbf{j}$ such that $\varphi_{\mathbf{i}}(0)=\varphi_{\mathbf{j}}(0)$. Applying $(2.2)$ and $\mathbf{i} \neq \mathbf{j}$, there exist $a_{i}, a_{i}^{\prime} \in\{0,1, s, t\}, 1 \leqslant i \leqslant n$ such that $a_{i_{0}} \neq a_{i_{0}}^{\prime}$ for some $1 \leqslant i_{0} \leqslant n$ and

$$
\sum_{i=1}^{n} a_{i} \beta^{i-1}=\sum_{i=1}^{n} a_{i}^{\prime} \beta^{i-1} .
$$

It follows that there are $L_{i}, L_{i}^{\prime} \in \mathcal{B}_{n}, i=1,2,3$ such that

$$
L_{1}+L_{2} s+L_{3} t=L_{1}^{\prime}+L_{2}^{\prime} s+L_{3}^{\prime} t .
$$

Furthermore, since each element of $\mathcal{B}_{n}$ has a unique representation, we conclude that

$$
\left(L_{1}, L_{2}, L_{3}\right) \neq\left(L_{1}^{\prime}, L_{2}^{\prime}, L_{3}^{\prime}\right) .
$$


Combining with $(2.3)$ we derive $\left(L_{2}, L_{3}\right) \neq\left(L_{2}^{\prime}, L_{3}^{\prime}\right)$. We claim that $L_{2} \neq L_{2}^{\prime}$ and $L_{3} \neq L_{3}^{\prime}$. Indeed assume to the contrary that $L_{2}=L_{2}^{\prime}$, then $L_{3} \neq L_{3}^{\prime}$ and hence

$$
t=\frac{L_{1}-L_{1}^{\prime}}{L_{3}^{\prime}-L_{3}} \in \mathbf{Q}_{\beta}
$$

which is contradict to the assumption that $t \notin \mathbf{Q}_{\beta}$. Similar argument yields $L_{3} \neq L_{3}^{\prime}$. Thus the above claim is true. Combining with (2.3) we obtain the desired identity.

2.3. Values of polynomials on algebraic numbers. Garsia [8, Lemma 1.51] first applied the estimates of polynomials with integer coefficients on algebraic numbers to the theory of Bernoulli convolution, since then this method and its variants have led to many applications in fractal geometry, see e.g. [7, Section 5], [9, Theorem 1.5], [13, Section 6], [16, 18].

The following form is taken from [16, Lemma 11]. Denote by $\mathcal{P}(n, H)$ the collection of integer coefficient polynomials with degree at most $n$ and its coefficients are bounded by $H$.

Lemma 2.3. For any algebraic number $\xi$ there exists $M>0$ depending only on $\xi$ such that for any $f \in \mathcal{P}(n, H)$ if $f(\xi) \neq 0$, then

$$
|f(\xi)| \geqslant M^{-n} H^{-M}
$$

We remark that one may obtain better lower bounds for some special algebraic numbers, for instance when $\beta$ is a Pisot number or Salem number, see [8, Lemma 1.51], [7, Section 5], [13, Section 6].

2.4. Continued fractions with non-integer letters. We start from recalling some well known facts from continued fractions, see [12] for more details. An expression of the form

$$
\frac{1}{a_{1}+\frac{1}{a_{2}+\frac{1}{a_{3}+\cdots \frac{1}{a_{k}}}}}
$$

is called a finite continued fraction, and for convenience we write it as

$$
\left[a_{1}, a_{2}, \ldots, a_{k}\right] \text {. }
$$

In general applications of continued fractions the letters $a_{1}, a_{2}, \ldots, a_{k}$ are often assumed to be positive integers. However, for our applications we always assume $a_{1}, a_{2}, \ldots, a_{k}$ to be elements of $\mathbf{P}_{\beta}$, and thus in general the elements $a_{1}, a_{2}, \ldots, a_{k}$ may not be natural numbers. i.e.,

Given a real number sequence $\left\{a_{n}\right\}$ with $a_{n} \geqslant 1$, denote $\frac{p_{k}}{q_{k}}$ the value of $(2.4)$,

$$
\frac{p_{k}}{q_{k}}=\frac{1}{a_{1}+\frac{1}{a_{2}+\frac{1}{a_{3}+\cdots \frac{1}{a_{k}}}} .}
$$

We remark that $p_{k}$ and $q_{k}$ are the "canonical representations" of the finite continued fractions, see [12, Chapter 1]. 
In the following we collect some useful facts for the sequence $\left\{\frac{p_{n}}{q_{n}}\right\}$ under the condition $a_{n} \geqslant 1$ for all $n \in \mathbf{N}$.

- For convenience denote $p_{0}=0, q_{0}=1$. For $k=2,3, \ldots$ we have

$$
\begin{aligned}
p_{k} & =a_{k} p_{k-1}+p_{k-2}, \\
q_{k} & =a_{k} q_{k-1}+q_{k-2} .
\end{aligned}
$$

- The sequence $\left\{\frac{p_{k}}{q_{k}}\right\}$ is convergent and we denote

$$
\left[a_{1}, a_{2}, \ldots, a_{n}, \ldots\right]=\lim _{k \rightarrow \infty} \frac{p_{k}}{q_{k}} .
$$

- Let $x:=\left[a_{1}, a_{2}, \ldots, a_{n}, \ldots\right]$. Then

$$
\frac{1}{q_{k}\left(q_{k+1}+q_{k}\right)}<\left|x-\frac{p_{k}}{q_{k}}\right|<\frac{1}{q_{k} q_{k+1}} .
$$

We remark that most of the above properties follow directly from [12, Chapter 1], but there is an exceptional case for the left hand side of the estimate (2.6) when the numbers $a_{n}$ are not integers. We show a simper proof in the following. We suppose that $p_{k} / q_{k}$ is at the left side of $x$, similar argument woks for the right side as well. By $[12$, p. 6] we have

$$
\frac{p_{k}}{q_{k}}<\frac{p_{k+2}}{q_{k+2}}<x .
$$

Combining with $[12$, p. 6] we derive

$$
\left|x-\frac{p_{k}}{q_{k}}\right|>\left|\frac{p_{k}}{q_{k}}-\frac{p_{k+2}}{q_{k+2}}\right|=\frac{a_{k+2}}{q_{k} q_{k+2}} .
$$

Applying (2.5) and the condition $a_{n} \geqslant 1$ we obtain

$$
q_{k+2} / a_{k+2} \leqslant q_{k+1}+q_{k} .
$$

Thus combining with (2.7) we obtain the desired lower bound in (2.6).

By [12, Theorem 14] if $\left\{a_{n}\right\}$ is a sequence of positive integers then $\left[a_{1}, a_{2}, \ldots\right]$ is an irrational number. We note that this property is used in [1] for showing the desired IFS does not have exact overlaps. For the sequence $\left\{a_{n}\right\}$ with $a_{n} \in \mathbf{P}_{\beta}$ we have the following substitution which plays a similar role to [1].

Lemma 2.4. Let $\beta \geqslant 2$ be an algebraic number and $\left\{a_{n}\right\}$ be a sequence with $a_{n} \in \mathbf{P}_{\beta}, n \in \mathbf{N}$. Denote $p_{k} / q_{k}=\left[a_{1}, \ldots, a_{k}\right]$ and consider $p_{k}, q_{k}$ as polynomials with indeterminate $\beta$. Suppose that $p_{k}, q_{k} \in \mathcal{P}\left(d_{k}, d_{k}\right)$ for some $d_{k} \in \mathbf{N}$ and

$$
a_{k+1} \geqslant M^{k d_{k}} d_{k}^{M}
$$

where $M$ is the same as in Lemma 2.3. Then $\left[a_{1}, a_{2}, \ldots\right] \notin \mathbf{Q}_{\beta}$.

Proof. Assume to the contrary that $\left[a_{1}, a_{2}, \ldots\right] \in \mathbf{Q}_{\beta}$. Then there exit $f(\beta), g(\beta) \in$ $\mathbf{Z}_{\beta}$ with $g(\beta) \neq 0$ such that

$$
\frac{f(\beta)}{g(\beta)}=\left[a_{1}, a_{2}, \ldots\right]
$$

For each $k \in \mathbf{N}$ by (2.5) and (2.6) we have

$$
0<\left|f(\beta) q_{k}-g(\beta) p_{k}\right|<g(\beta) / q_{k+1}<g(\beta) / a_{k+1} .
$$

Note that we can regard $f, g$ as elements of $\mathcal{P}(d, d)$ for some integer $d$. Combining with the condition $p_{k}, q_{k} \in \mathcal{P}\left(d_{k}, d_{k}\right)$, there exists $C>0$ such that

$$
f(\beta) q_{k}-g(\beta) p_{k} \in \mathcal{P}\left(C d_{k}, C d_{k}\right) .
$$


Applying Lemma 2.3 and the non-zero condition in (2.9), we obtain

$$
\left|f(\beta) q_{k}-g(\beta) p_{k}\right| \geqslant M^{-C d_{k}}\left(C d_{k}\right)^{-M}
$$

which is contradict to (2.8) and (2.9).

Remark 2.5. As we claimed before for some special algebraic numbers we have better lower bounds in Lemma 2.3, and hence we can obtain weak condition of $a_{k+1}$ in the above Lemma 2.4 for these special algebraic numbers.

Note that there is sequence $\left\{a_{n}\right\}$ with $a_{n} \in \mathbf{P}_{\beta}$ such that $\left[a_{1}, a_{2}, \ldots\right] \in \mathbf{Q}_{\beta}$. For instance for $a_{n}=\beta, n \in \mathbf{N}$ we have

$$
[\beta, \beta, \ldots]=\frac{1}{\beta+[\beta, \beta, \ldots]},
$$

and hence

$$
[\beta, \beta, \ldots]=\frac{\sqrt{\beta^{2}+4}-\beta}{2} .
$$

Suppose $\sqrt{\beta^{2}+4} \in \mathbf{Z}$ then $[\beta, \beta, \ldots] \in \mathbf{Q}_{\beta}$.

We remark that the best approximate property of continued fractions (with positive integer letters) is used in the construction of [1] for finding the desired parameters $s$ and $t$. The best approximate property [12, Chapter 2] claims that for any sequence of positive integers $\left\{a_{n}\right\}$, denote $s=\left[a_{1}, a_{2}, \ldots\right]$ and $p_{k} / q_{k}$ be its partial quotient, and for any integers $1 \leqslant q \leqslant q_{k}, p \in \mathbf{Z}$ one has

$$
\left|s-\frac{p}{q}\right| \geqslant\left|s-\frac{p_{k}}{q_{k}}\right| \text {. }
$$

Clearly, there is no such best approximate property for the general real number sequence $\left\{a_{n}\right\}$. However, if the sequence $\left\{a_{n}\right\}$ is a subset of $\mathbf{P}_{\beta}$ for some algebraic number $\beta$ then we have the following Lemma 2.6 which is sufficient for our application.

For $a_{1}, a_{2}, \ldots, a_{k} \in \mathbf{P}_{\beta}$ denote

$$
\mathcal{C}\left[a_{1}, a_{2}, \ldots, a_{k}\right]=\left\{\left[a_{1}, \ldots, a_{k}, a_{k+1}, \ldots\right]: a_{j} \in \mathbf{P}_{\beta}, j \geqslant k+1\right\} .
$$

Lemma 2.6. Let $a_{1}, \ldots, a_{k} \in \mathbf{P}_{\beta}$ and $n \in \mathbf{N}$. Then there exits $a_{k+1} \in \mathbf{P}_{\beta}$ such that for any $s \in \mathcal{C}\left[a_{1}, \ldots, a_{k+1}\right]$ and any $f, g \in \mathcal{P}(n, n)$ with $g(\beta) \neq 0$ we have

$$
\left|s-\frac{f(\beta)}{g(\beta)}\right| \geqslant c>0,
$$

where the constant $c$ depends on $a_{1}, \ldots, a_{k+1}$ and $n$. We stress that the constant $c$ will not depend on the specific choices of $f$ and $g$.

Proof. Denote $\frac{p_{k}}{q_{k}}=\left[a_{1}, \ldots, a_{k}\right]$. We proceed on a case-by-case basis depending on $\frac{p_{k}}{q_{k}}=\frac{f(\beta)}{g(\beta)}$ or not. First suppose that

$$
\frac{p_{k}}{q_{k}}=\frac{f(\beta)}{g(\beta)} .
$$

Then by (2.6) we obtain

$$
\left|s-\frac{f(\beta)}{g(\beta)}\right|=\left|s-\frac{p_{k}}{q_{k}}\right|>\frac{1}{q_{k}\left(q_{k+1}+q_{k}\right)},
$$

thus this gives a positive lower bound. 
We now turn to the case $\frac{p_{k}}{q_{k}} \neq \frac{f(\beta)}{g(\beta)}$. Together with (2.6) we obtain

$$
\left|s-\frac{f(\beta)}{g(\beta)}\right| \geqslant\left|\frac{p_{k}}{q_{k}}-\frac{f(\beta)}{g(\beta)}\right|-\left|s-\frac{p_{k}}{q_{k}}\right| \geqslant \frac{\left|p_{k} g(\beta)-q_{k} f(\beta)\right|}{\left|q_{k} g(\beta)\right|}-\frac{1}{q_{k} q_{k+1}} .
$$

By (2.5) we have $p_{k}, q_{k} \in \mathbf{Z}_{\beta}$ and we consider $p_{k}, q_{k}$ as polynomials. Therefore, there exists a large integer $L$ which depends on $a_{1}, \ldots, a_{k}, n$ such that

$$
p_{k} g(\beta)-q_{k} f(\beta) \in \mathcal{P}(L, L) .
$$

Clearly the condition $\frac{p_{k}}{q_{k}} \neq \frac{f(\beta)}{g(\beta)}$ implies $p_{k} g(\beta)-q_{k} f(\beta) \neq 0$. Thus by Lemma 2.3 there exits $M$ depending only on $\beta$ such that

$$
\left|p_{k} g(\beta)-q_{k} f(\beta)\right| \geqslant M^{-L} L^{-M} .
$$

Moreover, there exists $M_{1}$ depending on $a_{1}, \ldots, a_{k}, n$ such that

$$
q_{k} g(\beta) \leqslant M_{1} \text {. }
$$

Let $a_{k+1} \in \mathbf{P}_{\beta}$ be a large number such that $a_{k+1}>2 M^{L} L^{M} M_{1}$, then by (2.5) we have

$$
\left(q_{k} q_{k+1}\right)^{-1} \leqslant a_{k+1}^{-1} \leqslant M^{-L} L^{-M} M_{1}^{-1} / 2 .
$$

Combining with (2.11), (2.12) and (2.13), we obtain

$$
\left|s-\frac{f(\beta)}{g(\beta)}\right| \geqslant M^{-L} L^{-M} M_{1}^{-1} / 2 .
$$

Together with (2.10) we obtain the desired result.

Thanks to the referee for suggesting the following simper proof of Lemma 2.6 which is based on the pigeonhole principle. Since $\beta \geqslant 2$, for any fixed $a_{1}, \ldots, a_{k} \in \mathbf{P}_{\beta}$ the sets

$$
\overline{\mathcal{C}\left[a_{1}, \ldots, a_{k}, a_{k+1}\right]}, \quad a_{k+1} \in \mathbf{P}_{\beta}
$$

are pairwise disjoint. Note that $\mathbf{P}_{\beta}$ is infinite but for any $n$ the set $\mathcal{P}(n, n)$ is finite. This implies the existence of the required $a_{n+1} \in \mathbf{P}_{\beta}$ and constant $c>0$.

\section{Proofs of Theorem 1.2}

We mimick the construction of Baker [1] to our setting. Without loss of generality we assume that the sequence $\left\{\varepsilon_{n}\right\}$ is monotone decreasing. Otherwise we may consider the sequence $\left\{\varepsilon_{n}^{\prime}\right\}$ where $\varepsilon_{n}^{\prime}=\min _{1 \leqslant i \leqslant n} \varepsilon_{i}$.

Initial construction. Let $a_{1}=1$ then by the definition of continued fractions we have $p_{1}=q_{1}=1$. Thus $p_{1}, q_{1} \in \mathcal{B}_{1}$ where $\mathcal{B}_{1}$ is given as in (2.1). Let $N_{1} \geqslant 2$ a natural number then there exists $a_{2} \in \mathbf{P}_{\beta}$ such that for any $s \in \mathcal{C}\left[a_{1}, a_{2}\right]$, by (2.6), we have

$$
|s-1|=\left|q_{1} s-p_{1}\right|<1 / a_{2} \leqslant \varepsilon_{N_{1}} .
$$

Taking sufficiently large $a_{2} \in \mathbf{P}_{\beta}$ such that by (2.5) there exits $n \in \mathbf{N}$ with

$$
p_{2}, q_{2} \in \mathcal{B}_{n} .
$$

Moreover, by taking sufficiently large $a_{2} \in \mathbf{P}_{\beta}$ and applying Lemma 2.6, there exists a positive $c_{1}$ such that for any $f, g, h \in \mathcal{P}(1,1)$ with $g(\beta) \neq 0$ one has

$$
\left|s-\frac{f(\beta)}{g(\beta)}-\frac{h(\beta)}{g(\beta)}\right| \geqslant c_{1} \text {. }
$$


Let $a_{2} \in \mathbf{P}_{\beta}$ be sufficiently large such that the above (3.1), (3.2), and (3.3) hold. Denote

$$
N_{2}=\min \left\{n \in \mathbf{N}: p_{2}, q_{2} \in \mathcal{B}_{n}\right\} .
$$

Now we turn to the construction of $t$. Let $a_{1}^{\prime}=1$ then by the definition of continued fractions we have $p_{1}^{\prime}=q_{1}^{\prime}=1$. For $\varepsilon_{N_{2}}$ and $c_{1}$ there exists $a_{2}^{\prime} \in \mathbf{P}_{\beta}$ such that for any $t \in \mathcal{C}\left[a_{1}^{\prime}, a_{2}^{\prime}\right]$ we have

$$
|t-1|=\left|q_{1}^{\prime} t-p_{1}^{\prime}\right|<1 / a_{2}^{\prime} \leqslant \min \left\{\varepsilon_{N_{2}}, c_{1}\right\} .
$$

We remark that the above conditions (3.3), (3.5) and their inductive versions (3.9), (3.14) below will be used to prove that the final IFS $\Phi_{\beta, s, t}$ does not have exact overlaps.

Moreover, we ask that $a_{2}$ and $a_{2}^{\prime}$ are sufficiently large such that they satisfy the condition (2.8) of Lemma 2.4 with $d_{1}=1$ respectively. Denote

Taking sufficiently large $a_{2}^{\prime} \in \mathbf{P}_{\beta}$ such that there exits $n \in \mathbf{N}$ with $p_{2}^{\prime}, q_{n}^{\prime} \in \mathcal{B}_{n}$.

$$
M_{2}=\min \left\{n \in \mathbf{N}: p_{2}^{\prime}, q_{2}^{\prime} \in \mathcal{B}_{n}\right\} .
$$

Note that we start from the definition of $M_{2}$ other than $M_{1}$ for the convenience of our notation in the iterated construction below.

Iterated construction. Let $k \geqslant 2$. Suppose that there are $a_{2}, \ldots, a_{k}$ and $a_{2}^{\prime}, \ldots, a_{k}^{\prime}$ such that for any

$$
s \in \mathcal{C}\left[a_{1}, \ldots, a_{k}\right] \text { and } t \in \mathcal{C}\left[a_{1}^{\prime}, \ldots, a_{k}^{\prime}\right]
$$

we have

$$
\Delta_{n}(\beta, s, t) \leq \varepsilon_{n}, \quad \forall 1 \leqslant n \leqslant N_{k}
$$

Moreover,

$$
p_{k}, q_{k} \in \mathcal{B}_{N_{k}} \quad \text { and } \quad p_{k}^{\prime}, q_{k}^{\prime} \in \mathcal{B}_{M_{k}} .
$$

Note that the parameters $N_{2}, M_{2}$ are given at (3.4), (3.6) respectively. Furthermore there exists a positive number $c_{k-1}$ such that for any

$$
s \in \mathcal{C}\left[a_{1}, \ldots, a_{k}\right] \text { and } t \in \mathcal{C}\left[a_{1}^{\prime}, \ldots, a_{k-1}^{\prime}\right]
$$

and any $f, g, h \in \mathcal{P}(k-1, k-1)$ with $g(\beta) \neq 0$ we have

$$
\left|s-\frac{p_{k-1}^{\prime} f(\beta)}{q_{k-1}^{\prime} g(\beta)}-\frac{h(\beta)}{g(\beta)}\right| \geqslant c_{k-1},
$$

and

$$
\left|t-\frac{p_{k-1}^{\prime}}{q_{k-1}^{\prime}}\right| \leqslant \frac{c_{k-1}}{k-1} .
$$

Note that we may add further conditions $M_{k} \geqslant 2 N_{k}$ and $N_{k}>M_{k-1}$ to make sure that $N_{k}$ tends to infinity as $k$ tends to infinity. Moreover, we ask that $a_{k}$ and $a_{k}^{\prime}$ are large enough such that they satisfy the condition (2.8) of Lemma 2.4 respectively for some $d_{k} \in \mathbf{N}$ which admits $p_{k}, q_{k}, p_{k}^{\prime}, q_{k}^{\prime} \in \mathcal{P}\left(d_{k}, d_{k}\right)$.

Under the above assumption, we now begin to choose $a_{k+1}$ and $a_{k+1}^{\prime}$ such that the above claims still hold for the case $k+1$.

For the number $\varepsilon_{M_{k}}$ there exists $a_{k+1} \in \mathbf{P}_{\beta}$ such that for any

$$
s \in \mathcal{C}\left[a_{1}, \ldots, a_{k+1}\right]
$$

by (2.6) we have

$$
\left|q_{k} s-p_{k}\right| \leqslant 1 / a_{k+1} \leqslant \varepsilon_{M_{k}} .
$$


Combining with Lemma 2.1 and the condition (3.8) we derive that

$$
\Delta_{n}(\beta, s, t) \leqslant \beta^{-n}\left|q_{k} s-p_{k}\right| \leqslant \varepsilon_{M_{k}}, \quad \forall N_{k} \leqslant n \leqslant M_{k} .
$$

Applying (2.5) and the assumption $p_{k}, q_{k} \in \mathcal{B}_{N_{k}}$, and by taking some large enough $a_{k+1} \in \mathbf{P}_{\beta}$, there exists a natural number $n>N_{k}$ such that

$$
p_{k+1}, q_{k+1} \in \mathcal{B}_{n} .
$$

Furthermore, by Lemma 2.6 there exits $a_{k+1} \in \mathbf{P}_{\beta}$ such that for any

$$
s \in \mathcal{C}\left[a_{1}, \ldots, a_{k+1}\right],
$$

and any $f, g, h \in \mathcal{P}(k, k)$ with $g(\beta) \neq 0$ one has

$$
\left|s-\frac{f(\beta)}{g(\beta)} \frac{p_{k}^{\prime}}{q_{k}^{\prime}}-\frac{h(\beta)}{g(\beta)}\right| \geqslant c_{k}>0,
$$

where $c_{k}$ depends on $\beta, k$ and $a_{2}, \ldots, a_{k+1}, a_{2}^{\prime}, \ldots, a_{k}^{\prime}$.

We take large enough $a_{k+1} \in \mathbf{P}_{\beta}$ such that the above (3.10), (3.12) and (3.13) still hold with respect to $a_{k+1}$. Denote

$$
N_{k+1}=\min \left\{n \in \mathbf{N}: p_{k+1}, q_{k+1} \in \mathcal{B}_{n}\right\} .
$$

For $\varepsilon_{N_{k+1}}$ and $c_{k}$ there exists $a_{k+1}^{\prime} \in \mathbf{P}_{\beta}$ such that for any

$$
t \in \mathcal{C}\left[a_{1}^{\prime}, \ldots, a_{k+1}^{\prime}\right]
$$

by (2.6) we have

$$
\left|q_{k}^{\prime} t-p_{k}^{\prime}\right| \leqslant 1 / a_{k+1}^{\prime} \leqslant \min \left\{\varepsilon_{N_{k+1}}, c_{k} / k\right\} .
$$

Combining with Lemma 2.1 and the condition (3.8) we obtain

$$
\Delta_{n}(\beta, s, t) \leqslant \beta^{-n}\left|q_{k}^{\prime} t-p_{k}^{\prime}\right| \leqslant \varepsilon_{N_{k+1}}, \quad \forall M_{k} \leqslant n \leqslant N_{k+1} .
$$

Moreover, by (2.5) and the assumption $p_{k}^{\prime}, q_{k}^{\prime} \in \mathcal{B}_{M_{k}}$, by taking large enough $a_{k+1}^{\prime} \in \mathbf{P}_{\beta}$ we obtain $p_{k+1}^{\prime}, q_{k+1}^{\prime} \in \mathcal{B}_{n}$ for some $n \in \mathbf{N}$. Denote

$$
M_{k+1}=\min \left\{n \in \mathbf{N}: p_{k+1}^{\prime}, q_{k+1}^{\prime} \in \mathcal{B}_{n}\right\} .
$$

Furthermore, we ask that $a_{k+1}$ and $a_{k+1}^{\prime}$ are sufficiently large such that they satisfy the condition (2.8) of Lemma 2.4 respectively for some $d_{k+1} \in \mathbf{N}$ which admits $p_{k+1}, q_{k+1}, p_{k+1}^{\prime}, q_{k+1}^{\prime} \in \mathcal{P}\left(d_{k+1}, d_{k+1}\right)$.

By (3.7), (3.11) and (3.15) we derive that for any

$$
s \in \mathcal{C}\left[a_{1}, \ldots, a_{k+1}\right] \quad \text { and } \quad t \in \mathcal{C}\left[a_{1}^{\prime}, \ldots, a_{k+1}^{\prime}\right]
$$

we have

$$
\Delta_{n}(\beta, s, t) \leqslant \varepsilon_{n}, \quad \forall 1 \leqslant n \leqslant N_{k+1} .
$$

By iterating the above arguments, there exist two sequences $\left\{a_{k}\right\}$ and $\left\{a_{k}^{\prime}\right\}$ such that

$$
s=\left[a_{1}, \ldots, a_{n}, \ldots\right] \notin \mathbf{Q}_{\beta} \quad \text { and } \quad t=\left[a_{1}^{\prime}, \ldots, a_{n}^{\prime}, \ldots\right] \notin \mathbf{Q}_{\beta},
$$

and

$$
\Delta(\beta, s, t) \leqslant \varepsilon_{n}, \quad \forall n \in \mathbf{N} .
$$

Moreover for each $k \in \mathbf{N}$ and any $f, g, h \in \mathcal{P}(k, k)$ with $g(\beta) \neq 0$ we have

$$
\left|s-\frac{f(\beta)}{g(\beta)} \frac{p_{k}^{\prime}}{q_{k}^{\prime}}-\frac{h(\beta)}{g(\beta)}\right| \geqslant c_{k}>0,
$$


where $c_{k}$ depends on $\beta, k$ and $a_{2}, \ldots, a_{k+1}, a_{2}^{\prime}, \ldots, a_{k}^{\prime}$. Furthermore by (3.14) we obtain

$$
\left|t-\frac{p_{k}^{\prime}}{q_{k}^{\prime}}\right| \leq c_{k} / k
$$

No exact overlaps. The following arguments are due to [1]. For completeness we show the arguments here.

Let $s, t$ be as in the above construction. Assume to the contrary that $\Phi_{\beta, s, t}$ has exact overlap. Then by Lemma 2.2 there are $f(\beta), g(\beta) \in \mathbf{Z}_{\beta} \backslash\{0\}$ and $h(\beta) \in \mathbf{Z}_{\beta}$ such that

$$
s=\frac{f(\beta)}{g(\beta)} t+\frac{h(\beta)}{g(\beta)} .
$$

Combining with (3.17), for each $k$ we have

$$
\left|s-\frac{f(\beta)}{g(\beta)} \frac{p_{k}^{\prime}}{q_{k}^{\prime}}-\frac{h(\beta)}{g(\beta)}\right| \leqslant\left|\frac{f(\beta)}{g(\beta)}\right|\left|t-\frac{p_{k}^{\prime}}{q_{k}^{\prime}}\right| \leqslant \frac{c_{k}|f(\beta)|}{k|g(\beta)|} .
$$

Other the other hand, we could consider $f(\beta), g(\beta), h(\beta)$ as polynomials and they belongs to $\mathcal{P}(k, k)$ for all large enough $k$. Therefore, by (3.16) we obtain

$$
\left|s-\frac{f(\beta)}{g(\beta)} \frac{p_{k}^{\prime}}{q_{k}^{\prime}}-\frac{h(\beta)}{g(\beta)}\right| \geqslant c_{k},
$$

which contradicts (3.18) when $k$ is large enough.

Acknowledgement. It is my pleasure to thank the members of the online reading group on fractal geometry in the department of mathematics. In particular, I am grateful to De-Jun Feng for the very careful reading of the manuscript and many valuable suggestions. The author thanks Demi Allen and Simon Baker for many valuable comments. The author also would like to thank the anonymous referee for the very careful reading of the manuscript and many helpful comments. This work was supported by HKRGC GRF Grants CUHK14301218 and CUHK14304119.

\section{References}

[1] BAKER, S.: Iterated function systems with super-exponentially close cylinders. - Adv. Math. $379,2021,107548$.

[2] BAKER, S.: Iterated function systems with super-exponentially close cylinders II. - Preprint, arxiv.org/abs/2007.11291.

[3] BÁRÁNy, B., and A. KÄENMÄKI: Superexponential condensation without exact overlaps. Preprint, arxiv.org/abs/1910.04623.

[4] BARral, J., and D.-J. Feng: On multifractal formalism for self-similar measures with overlaps. - Math. Z. 298, 2021, 359-383.

[5] Falconer, K. J.: Dimensions and measures of quasi self-similar sets. - Proc. Amer. Math. Soc. 106:2, 1989, 543-554.

[6] FAlconer, K. J.: Fractal geometry: Mathematical foundations and applications. Second Edition. - John Wiley, NJ, 2003.

[7] FenG, D.-J.: Gibbs properties of self-conformal measures and the multifractal formalism. Ergodic Theory Dynam. Systems 27:3, 2007, 787-812.

[8] Garsia, A. M.: Arithmetic properties of Bernoulli convolutions. - Trans. Amer. Math. Soc. 102, 1962, 409-432.

[9] Hochman, M.: On self-similar sets with overlaps and inverse theorems for entropy. - Ann. of Math. (2) 180:2, 2014, 773-822. 
[10] Hochman, M.: Dimension theory of self-similar sets and measures. - Proceedings of the International Congress of Mathematicians, Volume II, Rio de Janeiro, 2018, 1943-1966.

[11] Hutchinson, J. E.: Fractals and self-similarity. - Indiana Univ. Math. J. 30:5, 1981, 713-747.

[12] Khinchin, A. Ya.: Continued fractions. - The University of Chicago Press, Chicago, Ill. London, 1964.

[13] LAU, K.-S., and S.-M. NGAI: Multifractal measures and a weak separation condition. - Adv. Math. 141:1, 1999, 45-96.

[14] Mattila, P.: Fourier analysis and Hausdorff dimension. - Cambridge Stud. Adv. Math. 50, Cambridge Univ. Press, 2015.

[15] NGai, S.-M., and Y. WANG: Hausdorff dimension of self-similar sets with overlaps. - J. Lond. Math. Soc. (2) 63, 2001, 655-672.

[16] RAPAPORT, A.: Proof of the exact overlaps conjecture for systems with algebraic contractions. - Preprint, arxiv.org/abs/2001.01332.

[17] Simon, K.: Overlapping cylinders: the size of a dynamically defined Cantor set. - In: Ergodic theory of $\mathbf{Z}^{d}$ actions (Warwick, 1993-1994), London Math. Soc. Lecture Note Ser. 228, 1996, 259-272.

[18] VArJú, P. P.: On the dimension of Bernoulli convolutions for all transcendental parameters. - Ann. of Math. (2) 189:3, 2019, 1001-1011.

Received 2 May 2020 • Accepted 13 October 2020 